\title{
Scattering of Toroidal Waves by Semitransparent Reflectors of Revolution in Application to Omnidirectional Antennas
}

\author{
Kirill Klionovski
}

\begin{abstract}
Problems of radiation pattern synthesis, sidelobe suppression, or back radiation suppression appear when using antennas with reflectors or ground planes. The use of a semitransparent surface allows the required shape of a radiation pattern to be synthesized efficiently through the variation of reflection and transmission coefficients. Effective radiation pattern synthesis is possible when using analytical formulae designed for the radiation patterns of antennas with semitransparent reflectors. In this paper, we present a model for the scattering of a toroidal wave of a general form by means of a semitransparent reflector of revolution. The model allowed us to simulate the radiation patterns of various kinds of axisymmetric antennas with semitransparent reflectors and ground planes. Two asymptotic expansions of the radiation pattern of the model were found in the physical optics approximation. The expansions allow the radiation pattern for the entire space to be determined. As an example of its application, we used the model to calculate the radiation patterns of a plane with Archimedean two-wire spiral antenna and a loop antenna above concave and convex reflectors, as well as an Archimedean slot spiral antenna with a ground plane. We synthesized the optimal profiles of perfectly conducting and semitransparent reflectors and ground planes to improve the front-to-back ratio of the antennas.
\end{abstract}

Index Terms-Toroidal wave, semitransparent reflector, physical optics approximation, Archimedean spiral antenna, loop antenna.

\section{INTRODUCTION}

$\mathrm{T}$ HE use of semitransparent surfaces is a novel trend in antenna engineering for applications in which special shapes of radiation patterns are required. Semitransparent ground planes provide back radiation suppression of omnidirectional antennas [1-4]. Reflectors with semitransparent edges provide a desirable sidelobe level and reduce a backscattering mainlobe $[5,6]$. Semitransparent disk ground planes and reflectors of revolution are often used with axisymmetric antennas, such as loop antennas, circular patch antennas, monopoles, or conical horns. The optimization of the transparency distribution on the surface of a semitransparent reflector is the main issue in the synthesis procedure for obtaining the required shape of a radiation pattern. Therefore, a simple model that allows the radiation patterns of various

K. Klionovski is with the King Abdullah University of Science and Technology (KAUST), Thuwal, 23955-6900, Kingdom of Saudi Arabia (email: kirill.klionovski@kaust.edu.sa). omnidirectional antennas with semitransparent reflectors to be calculated using analytical formulae is useful for the optimization issue.

The radiation patterns of omnidirectional antennas in the absence of a reflector can be calculated using simple approximations of toroidal wave sources. For example, the radiation patterns of a loop antenna and a plane with an Archimedean two-wire spiral antenna working at the first harmonic radiation mode coincide with the radiation patterns of the annular electric current of a traveling wave [2, 7]. The radiation pattern of a circular patch antenna coincides with the radiation pattern of an annular magnetic current with a single azimuthal variation [8]. The radiation pattern of a monopole can be calculated as the sum of the radiation patterns of a set of discrete annular in-phase axial electric currents [2]. The radiation of the $\mathrm{TE}_{11}$ mode of an open-ended circular waveguide is a toroidal wave with a single azimuthal variation, while the radiation of a conical or coaxial horn can be expressed as the superposition of these toroidal waves [9]. Thus, a model of the scattering of the field of a toroidal wave by a semitransparent reflector of the revolution allows the radiation patterns of various types of omnidirectional antennas to be found using reflectors.

Some particular cases of a toroidal wave scattering by perfectly electrically conducting (PEC) and semitransparent reflectors are investigated in [1, 2, 10-20]. In particular, the scattering of a field induced by an annular current on a PEC disk is investigated in [10-15]. Some asymptotic solutions for a scalar toroidal wave scattering by the PEC concave surface of a sphere are considered in [16-18]. A numerical solution for the scattering of an in-phase azimuthal annular electric current field by a PEC conical reflector is considered in [19]. A scalar toroidal wave scattering by a PEC bi-conical reflector is considered in [20], within which the first two terms of a nonuniform asymptotic expansion of a radiation pattern are obtained. The scattering of a field induced by an annular magnetic current with a single azimuthal harmonic on a semitransparent disk is investigated in [1] using the numerical solution of an integral equation by the method of moments (MoM). Two-term asymptotic expansions of the radiation pattern of a toroidal wave scattered by a semitransparent disk 
are available in [2].

In this paper, we considered the problem of the scattering of the field of a toroidal (particularly spherical) wave of a general form by a semitransparent (particularly PEC) arbitrarily shaped reflector of revolution. We used the physical optics (PO) approximation and the stationary phase method to obtain asymptotic formulae for the calculation of the radiation pattern of the considered problem. We used the asymptotic formulae to optimize the shape of a PEC reflector and the transparency of a semitransparent reflector for the purpose of the back radiation suppression of wire and slot Archimedean spiral antennas and a loop antenna. To verify the optimization results, we conducted a numerical simulation of these antennas using a MoM-based electromagnetic solver.

\section{STATEMENT Of ThE TASK}

\section{A. The Geometry of the Task}

If we consider the scattering of a toroidal wave with harmonic azimuthal dependence by a semitransparent reflector, this kind of wave is excited by an annular current of radius $a$ with a dependence of $\mathbf{j}(z) \cos (n \varphi+\psi)$ in the cylindrical coordinates $(\rho, \varphi, z)$. The dimension of the annular current along the $z$-axis is $\Delta h$. The current is located at the distance $h$ from the center of the reflector (Figs. 1 (a)-(d)). The center of the reflector is located at the origin of the coordinates. The reflector is the surface of revolution relative to the $z$-axis. The axis of the annular current coincides with the axis of the reflector. A smooth function $z=f(\rho), \rho \in[0, L]$, determines the shape of the reflector. We assume that $f(\rho)$ is a slowly varying function at a distance equal to the wavelength. It has a finite derivative $f^{\prime}(\rho)=d(f(\rho)) / d \rho$. The derivative $f^{\prime}(\rho)$ can be positive (concave reflector (Fig. 1 (a),(b))), negative (convex reflector (Fig. 1 (c))), or change the sign in the interval $[0, L]$ (concavo-convex reflector (Fig. $1(\mathrm{~d}))$ ). In a general case, $f^{\prime}(\rho)$ can change the sign in the interval $[0, L]$ several times. We assumed that each point of the source of the toroidal wave fully illuminates the surface of the reflector, and there is no multiple diffraction by the surface of the reflector for a reflected ray in the geometrical optics (GO) approximation.

\section{B. The Toroidal Wave}

The vector potential of the annular source of the toroidal wave in the free space is determined by integrating the product of the annular current and the Green function [2]:

$$
\begin{aligned}
& \mathbf{A}(\mathbf{r})=\frac{a}{4 \pi} \int_{0}^{2 \pi} \cos \left(n \varphi^{\prime}+\psi\right) \int_{0}^{\Delta h} \mathbf{j}\left(z^{\prime}\right) \frac{e^{-i k\left|\mathbf{r}-\mathbf{r}^{\prime}\right|}}{\left|\mathbf{r}-\mathbf{r}^{\prime}\right|} d z^{\prime} d \varphi^{\prime} \\
& \left|\mathbf{r}-\mathbf{r}^{\prime}\right|=\left(\left(\rho \cos \varphi-a \cos \varphi^{\prime}\right)^{2}+\right. \\
& \left.+\left(\rho \sin \varphi-a \sin \varphi^{\prime}\right)^{2}+\left(z-z^{\prime}\right)^{2}\right)^{\frac{1}{2}}
\end{aligned}
$$

Here, $\mathbf{r}(\rho, \varphi, z)$ is the position vector passing from the origin of the coordinates to an observation point; $\mathbf{r}^{\prime}\left(\rho^{\prime}, \varphi^{\prime}, z^{\prime}\right)$ is the position vector passing from the origin of the coordinates to a source point; $n=0,1,2, \ldots ; \psi$ is the initial phase; $k=2 \pi / \lambda ; \lambda$ is
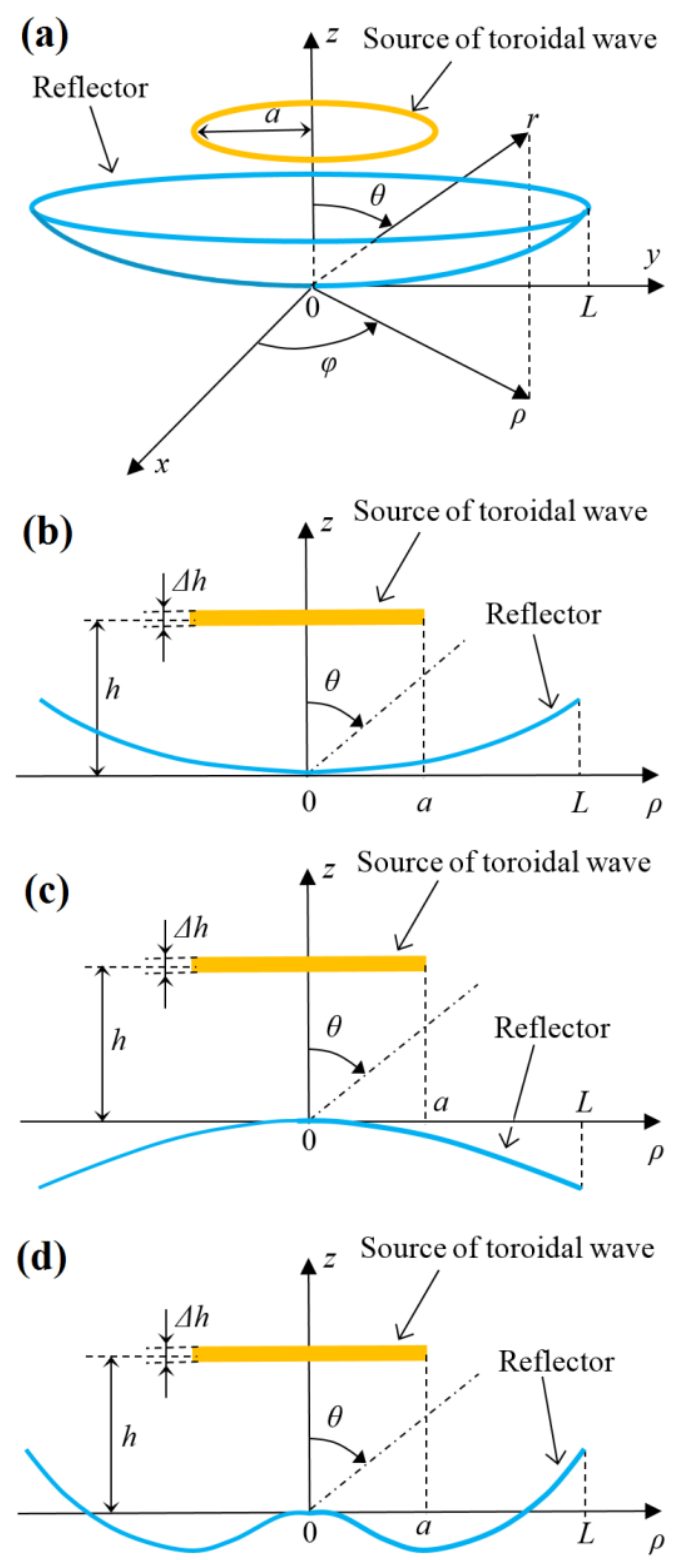

Fig. 1. (a) the general view of the source of the toroidal wave above the concave reflector; (b) the side view of the source above the concave reflector; (c) the side view of the source above the convex reflector; (d) the side view of the source above the concavo-convex reflector.

the radiation wavelength in vacuum; and $i$ is the imaginary unit. The radiation patterns of the meridional $\mathrm{H}_{\theta}(\theta, \varphi)$ and azimuthal $\mathrm{H}_{\varphi}(\theta, \varphi)$ components of the magnetic field intensity vector of the toroidal wave in the spherical coordinates $(r, \theta, \varphi)$ in the free space are calculated through the vector potential (1). Applying the stationary phase method to the exterior integral in (1) when the stationary point was far from the end of the integration [21], we obtained the following asymptotic expression of the vector potential:

$$
\begin{aligned}
& \mathbf{A}(\mathbf{r})=\frac{\sqrt{a} \cos (n \varphi+\psi)}{4 \sqrt{2 \pi k \rho}} \times \\
& \times \sum_{m=0}^{1}(-1)^{m n} \int_{0}^{\Delta h} \mathbf{j}\left(z^{\prime}\right) \frac{e^{-i k\left|\mathbf{r}-\mathbf{r}_{m}{ }^{\prime}\right|-(-1)^{m} i \frac{\pi}{4}}}{\sqrt{\left|\mathbf{r}-\mathbf{r}_{m}{ }^{\prime}\right|}} d z^{\prime},
\end{aligned}
$$


where $\mathbf{r}_{m}{ }^{\prime}=\mathbf{r}^{\prime}\left(a, \varphi+m \pi, \mathbf{z}^{\prime}\right)$. Expression (2) is valid when an observation point is far from the $z$-axis. When the width of the annular current is much smaller that its radius (i.e., $\Delta h \ll<a$ ), the vector potential can be simplified:

$$
\begin{aligned}
& \mathbf{A}(r, \theta, \varphi)=\frac{\sqrt{a} \cos (n \varphi+\psi)}{4 \sqrt{2 \pi k r \sin \theta}} \mathbf{D}(\theta) \times \\
& \times \sum_{m=0}^{1}(-1)^{m n} \frac{e^{-i k\left(r-(-1)^{m} a \sin \theta\right)-(-1)^{m} i \frac{\pi}{4}}}{\sqrt{r-(-1)^{m} a \sin \theta}}, \\
& \mathbf{D}(\theta)=\int_{0}^{\Delta h} \mathbf{j}\left(z^{\prime}\right) e^{i k z^{\prime} \cos \theta} d z^{\prime} .
\end{aligned}
$$

Here, $\mathbf{D}(\theta)$ is the radiation pattern of the differential element of the annular current in the meridional plane. Thus, within the term "a toroidal wave," we consider any wave that is created by the vector potential in the form (3) for observation points far from the axis of a source. Analysis of the term $r \pm a \sin \theta$ of the sum in (3) showed that the radiation of the toroidal wave is created by the two points $Q_{1,2}$ located in one $z \rho$-plane at opposite ends of the source (Fig. 2) when the observation points are far from the axis of the source. The radiation from the opposite ends of the source leads to the appearance of the factor $\mathbf{J}_{n-1}(k a \sin \theta) \pm \mathbf{J}_{n+1}(k a \sin \theta)$ in the expression of the radiation pattern of the annular source. Here, $\mathbf{J}_{n}(k a \sin \theta)$ is the Bessel function of order $n$ and $\operatorname{argument} k a \sin \theta$. Thus, in accordance with (3), the radiation pattern of the source in the meridional plane is proportional to the production of the pattern $\mathbf{D}(\theta)$ and

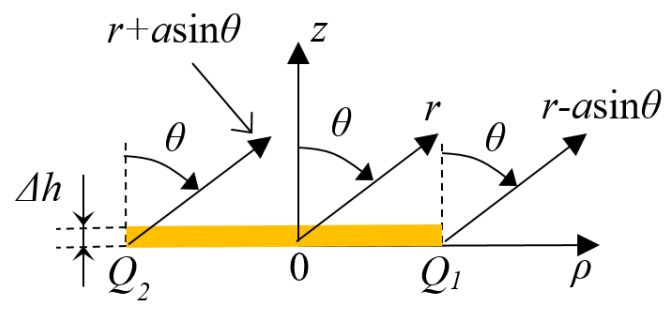

Fig. 2. Radiation from the opposite ends of the annular source.

the sum or difference of the Bessel functions. The radiation patterns of toroidal waves in the particular cases of the ring sources of radial, azimuthal, and axial electric and magnetic currents are presented in the appendix.

It should be noted that a toroidal wave becomes a spherical wave when the radius of the source tends to zero $(a \rightarrow 0)$. In this case, the annular source becomes a point source. Therefore, the theory presented in this paper for annular sources of toroidal waves is also applicable to dipole sources of spherical waves.

\section{The Boundary Conditions}

The infinitely thin semitransparent reflector is generally characterized by two reflection coefficients $\mathrm{R}_{\tau, \varphi}$, and two transmission coefficients $\mathrm{T}_{\tau, \varphi}=1-\mathrm{R}_{\tau, \varphi}$ (for waves with tangential $(\tau)$ and azimuthal $(\varphi)$ components of the magnetic field intensity vector on the surface of the reflector). A unit tangential vector $\tau_{0}$ on the surface of the reflector is calculated using the unit cylindrical radial $\boldsymbol{\rho}_{0}$ and axial $\mathbf{z}_{0}$ vectors as follows: $\boldsymbol{\tau}_{0}(\rho)=\boldsymbol{\rho}_{0} \cos \left(\theta^{\prime}(\rho)\right)+\mathbf{z}_{0} \sin \left(\theta^{\prime}(\rho)\right)$, where $\theta^{\prime}(\rho)=\arctan \left(f^{\prime}(\rho)\right)$. In the axisymmetric case, the reflection and transmission coefficients only depend upon radial coordinate $\rho$ and the angle of incidence of a wave incident on the reflector at the point $z=f(\rho)$. The boundary conditions on the surface of the semitransparent reflector are the following:

1. The tangential and azimuthal components of an electric field intensity vector are continual: $\left(\mathbf{n} \times \mathbf{E}^{+}\right)-\left(\mathbf{n} \times \mathbf{E}^{-}\right)=0$. Here $\mathbf{E}^{+}$and $\mathbf{E}^{-}$is the electric field intensity vector on the illuminated and shady surface of the reflector, respectively; $\mathbf{n}=\left(\boldsymbol{\tau}_{0} \times \boldsymbol{\varphi}_{0}\right)$ is the unit vector in the direction of the normal to the illuminated surface, while $\varphi_{0}$ is a unit azimuthal vector.

2. The tangential and azimuthal components of a magnetic field intensity vector have a discontinuity, which is equal to the electric current on the surface of the reflector $\mathbf{j}^{\mathrm{e}}$ : $\left(\mathbf{n} \times \mathbf{H}^{+}\right)-\left(\mathbf{n} \times \mathbf{H}^{-}\right)=\mathbf{j}^{\mathrm{e}}$. Here $\mathbf{H}^{+}$and $\mathbf{H}^{-}$is the magnetic field intensity vector on the illuminated and shady surface of the reflector, respectively.

3. An impedance tensor $\mathbf{Z}$ associates the tangential and azimuthal components of the electric field intensity vector with the electric current on the surface of the reflector: $\left(\mathbf{n} \times\left(\mathbf{n} \times \mathbf{E}^{+}\right)\right)=-\mathbf{Z} \mathbf{j}^{\mathrm{e}}$. The first condition can be written for the components of $\mathbf{E}^{+}$and $\mathbf{j}^{\mathrm{e}}$ in the following form:

$$
\left(\begin{array}{c}
E_{\tau}^{+} \\
E_{\varphi}^{+}
\end{array}\right)=\left(\begin{array}{ll}
Z_{\tau \tau} & Z_{\varphi \tau} \\
Z_{\tau \varphi} & Z_{\varphi \varphi}
\end{array}\right)\left(\begin{array}{c}
j_{\tau}^{e} \\
j_{\varphi}^{e}
\end{array}\right)
$$

where respectively $E_{\tau}{ }^{+}$and $E_{\varphi}{ }^{+}$are the tangential and azimuthal components of $\mathbf{E}^{+} ; j_{\tau}{ }^{e}$ and $j_{\varphi}{ }^{e}$ are respectively the tangential and azimuthal components of $\mathbf{j}$; and $Z_{\tau \tau}, Z_{\varphi \tau}, Z_{\tau \varphi}$, and $Z_{\varphi \varphi}$ are the components of the impedance tensor. In general, these tensor components are complex numbers argued to be within the range of $[-\pi / 2, \pi / 2]$.

\section{The Kirchhoff Integral}

The PO method was successfully used in [2] to determine the radiation pattern of a toroidal wave scattered by a semitransparent disk. Therefore, we used the PO method to determine the radiation pattern of a toroidal wave scattered by a semitransparent reflector of revolution. In accordance with the PO method and the boundary conditions, we assumed that there was no interaction between $j_{\tau}{ }^{e}$ and $j_{\varphi}{ }^{e}$, that is, $Z_{\varphi \tau}=Z_{\tau \varphi}=0$. The other components of the impedance tensor were associated with the reflection and transmission coefficients as follows:

$Z_{\tau \tau}=\frac{Z_{0}\left(1-\mathrm{R}_{\varphi}\right)}{2 \mathrm{R}_{\varphi}}, Z_{\varphi \varphi}=\frac{Z_{0}\left(1-\mathrm{R}_{\tau}\right)}{2 \mathrm{R}_{\tau}}$

Here, $Z_{0}=120 \pi \Omega$ is the free space wave impedance. The components of the electric current on the surface of the semitransparent reflector in the PO approximation were determined as follows:

$$
\left(\begin{array}{l}
j_{\tau}^{e} \\
j_{\varphi}^{e}
\end{array}\right)=\left(\begin{array}{cc}
0 & -2 \mathrm{R}_{\varphi} \\
2 \mathrm{R}_{\tau} & 0
\end{array}\right)\left(\begin{array}{l}
H_{\tau}^{\text {surf }} \\
H_{\varphi}^{\text {surf }}
\end{array}\right),
$$

where $H_{\tau}^{\text {surf }}$ and $H_{\varphi}^{\text {surf }}$ are respectively the tangential and 
azimuthal components of the magnetic field intensity vector of the toroidal wave on the surface of the reflector, which is determined using the vector potential (1) in free space.

We applied the PO method to the problem of the scattering of the toroidal wave created by the annular source, which is located above the semitransparent reflector. In the PO approximation, the meridional $H_{\theta}{ }^{P O}$ and azimuthal $H_{\varphi}{ }^{P O}$ components of the radiation pattern of the magnetic field intensity vector of the considered problem are expressed through the following Kirchhoff integrals:

$$
\begin{aligned}
& H_{\theta}^{P O}(\theta, \varphi)=H_{\theta}(\theta, \varphi) e^{i k h \cos \theta}+ \\
& +\int_{0}^{L} j_{\tau}^{e}\left(\rho, \varphi-\frac{\pi}{2 n}, \mathrm{z}=f(\rho)\right) H_{\theta}^{\tau}(\theta, \rho) e^{i k f(\rho) \cos \theta} d \rho+ \\
& +\int_{0}^{L} j_{\varphi}^{e}(\rho, \varphi, \mathrm{z}=f(\rho)) H_{\theta}^{\varphi}(\theta, \rho) e^{i k f(\rho) \cos \theta} d \rho, \\
& H_{\varphi}^{P O}(\theta, \varphi)=H_{\varphi}(\theta, \varphi) e^{i k h \cos \theta}+ \\
& +\int_{0}^{L} j_{\tau}^{e}(\rho, \varphi, \mathrm{z}=f(\rho)) H_{\varphi}^{\tau}(\theta, \rho) e^{i k f(\rho) \cos \theta} d \rho+ \\
& +\int_{0}^{L} j_{\varphi}^{e}\left(\rho, \varphi-\frac{\pi}{2 n}, \mathrm{z}=f(\rho)\right) H_{\varphi}^{\varphi}(\theta, \rho) e^{i k f(\rho) \cos \theta} d \rho .
\end{aligned}
$$

Here,

$$
\begin{aligned}
& H_{\theta}^{\tau}(\theta, \rho)=-i^{n} \frac{k \rho}{8 \pi} \cos \left(\theta^{\prime}(\rho)\right) \times \\
& \times\left[\int_{-\pi}^{\pi} e^{i\left(k \rho \sin \theta \sin \phi^{\prime}-(n-1) \phi^{\prime}\right)} d \phi^{\prime}+\int_{-\pi}^{\pi} e^{i\left(k \rho \sin \theta \sin \phi^{\prime}-(n+1) \phi^{\prime}\right)} d \phi^{\prime}\right] \\
& H_{\theta}^{\varphi}(\theta, \rho)=i^{n} \frac{k \rho}{8 \pi} \times \\
& \times\left[\int_{-\pi}^{\pi} e^{i\left(k \rho \sin \theta \sin \phi^{\prime}-(n-1) \phi^{\prime}\right)} d \phi^{\prime}-\int_{-\pi}^{\pi} e^{i\left(k \rho \sin \theta \sin \phi^{\prime}-(n+1) \phi^{\prime}\right)} d \phi^{\prime}\right] \\
& H_{\varphi}^{\tau}(\theta, \rho)=-i^{n} \frac{k \rho}{8 \pi} \cos \theta \cos \left(\theta^{\prime}(\rho)\right) \times \\
& \times\left[\int_{-\pi}^{\pi} e^{i\left(k \rho \sin \theta \sin \phi^{\prime}-(n-1) \phi^{\prime}\right)} d \phi^{\prime}-\int_{-\pi}^{\pi} e^{i\left(k \rho \sin \theta \sin \phi^{\prime}-(n+1) \phi^{\prime}\right)} d \phi^{\prime}\right]+ \\
& +i^{n+1} \frac{k \rho}{4 \pi} \sin \theta \sin \left(\theta^{\prime}(\rho)\right) \int_{-\pi}^{\pi} e^{i\left(k \rho \sin \theta \sin \phi^{\prime}-n \phi^{\prime}\right)} d \phi^{\prime}, \\
& H_{\varphi}^{\varphi}(\theta, \rho)=-i^{n} \frac{k \rho}{8 \pi} \cos \theta \times \\
& \times\left[\int_{-\pi}^{\pi} e^{i\left(k \rho \sin \theta \sin \phi^{\prime}-(n-1) \phi^{\prime}\right)} d \phi^{\prime}+\int_{-\pi}^{\pi} e^{i\left(k \rho \sin \theta \sin \phi^{\prime}-(n+1) \phi^{\prime}\right)} d \phi^{\prime}\right]
\end{aligned}
$$

The first term of the sums in (7) is the radiation pattern of the toroidal wave in free space; the second term is the radiation pattern of the tangential component of the electric current on the surface of the reflector; and the third term is the radiation pattern of the azimuthal component of the electric current on the surface of the reflector. The meridional $H_{\theta}{ }^{\tau(\varphi)}$ and azimuthal $H_{\varphi}{ }^{\tau(\varphi)}$ components from (8) are the radiation patterns in the meridional plane of a differential annular tangential (azimuthal) electric current of radius $\rho$ on the surface of the reflector. They were calculated through the integration of the Green function along the azimuthal coordinate in the interval $[-\pi, \pi]$.

\section{ASYMPTOTIC FORMULAE IN THE PHYSICAL OPTICS APPROXIMATION}

We assumed that the reflection and transmission coefficients were slowly varying functions, and the number of azimuthal harmonics was much smaller than the electric size of the source (i.e., $n<k a$ ). The last assumption is justified since the vast majority of axisymmetric antennas work at the first azimuthal harmonic radiation mode. We found the asymptotic expansions of the integrals (7) and (8) using the stationary phase method. Because this method does not allow a uniform asymptotic expansion for all angles $\theta$ to be obtained, we considered two separate cases: the intervals of angles $\theta$ near to or far from the $z$-axis.

\section{A. The Observation Angles Near the Z-Axis}

We used the stationary phase method when the stationary point was far from the end of the integration [21] for the interval of observation angles $\theta$ near the $z$-axis. In this case, we separately calculated the contributions of the stationary point and the end of the integration into the asymptotic expansions of the integrals (7). As a result, we obtained the following twoterm asymptotic expansion of the total field near the $z$-axis for the meridional (azimuthal) polarization of the incident wave:

$H_{\theta(\varphi)}^{P O}(\theta, \varphi)=H_{\theta(\varphi)}^{G O}(\theta, \varphi)+H_{\theta(\varphi)}^{s}(\theta, \varphi)$,

where $H_{\theta}{ }^{G O}$ and $H_{\varphi}{ }^{G O}$ are respectively the meridional and azimuthal components of the radiation pattern in the GO approximation, and $H_{\theta}{ }^{s}$ and $H_{\varphi}{ }^{s}$ are respectively the meridional and azimuthal components of the radiation pattern created by the electric current on the edge of the reflector at the point $z=f(L)$. The first and the second term of the sum in (9) correspond to the contribution of the stationary point and the end of the integration in the asymptotic expansion of the integral (7), respectively. The meridional (azimuthal) component of the GO pattern was calculated as follows:

$$
\begin{aligned}
& H_{\theta(\varphi)}^{G O}(\theta, \varphi)=H_{\theta(\varphi)}(\theta, \varphi) e^{i k s^{i n c}(\theta)} \chi\left(\theta^{i n c}-\theta\right)+ \\
& +\xi_{\theta(\varphi)} \mathrm{R}_{\tau(\varphi)}\left(\rho_{R}\right) H_{\theta(\varphi)}\left(\pi-\left(\theta+2 \theta^{\prime}\left(\rho_{R}\right)\right), \varphi\right) \times \\
& \times e^{i k s^{r e f l}(\theta)} \chi\left(\theta^{r e f l}-\theta\right)+ \\
& +\mathrm{T}_{\tau(\varphi)}\left(\rho_{T}\right) H_{\theta(\varphi)}(\theta, \varphi) e^{i k s^{i n c}(\theta)} \chi\left(\theta-\theta^{i n c}\right),
\end{aligned}
$$

where $\xi_{\theta(\varphi)}$ is equal to -1 and 1 for the meridional and azimuthal components of the radiation pattern, respectively: 


$$
\begin{aligned}
& \chi(\theta)=\left\{\begin{array}{ll}
1, & \theta \geq 0 \\
0, & \theta<0
\end{array} ; \theta^{i n c}=\arccos \left(\frac{f(L)-h}{L_{s e}}\right) ;\right. \\
& \theta^{r e f l}=\arccos \left(\frac{h-f(L)}{L_{s e}}\right)-2 \theta^{\prime}(L) ; \\
& L_{s e}=\sqrt{L^{2}+(h-f(L))^{2}} ; \quad s^{\text {inc }}(\theta)=h \cos \theta ; \\
& s^{\text {refl }}(\theta)= \begin{cases}\Delta_{\rho}^{m}\left(\rho_{R}\right) \sin \theta-\Delta_{z}^{m}\left(\rho_{R}\right) \cos \theta, \quad 0 \leq \theta \leq \theta^{\text {refl }} \\
\Delta_{\rho}^{m}(L) \sin \theta-\Delta_{z}^{m}(L) \cos \theta, \quad \theta^{\text {refl }}<\theta<\theta^{\text {inc }} ; \\
\Delta_{\rho}^{m}\left(\rho_{T}\right) \sin \theta-\Delta_{z}^{m}\left(\rho_{T}\right) \cos \theta, \quad \theta^{\text {inc }} \leq \theta \leq \pi\end{cases} \\
& \Delta_{\rho}^{m}(\rho)=\left(h+f(\rho)-\rho f^{\prime}(\rho)\right) \sin \left(2 \theta^{\prime}(\rho)\right) ; \\
& \Delta_{z}^{m}(\rho)=2\left(h+f(\rho)-\rho f^{\prime}(\rho)\right) \cos ^{2}\left(\theta^{\prime}(\rho)\right)-h .
\end{aligned}
$$

The following non-linear equations

$$
\begin{aligned}
& \frac{h-f\left(\rho_{R}\right)}{\sqrt{\rho_{R}^{2}+\left(h-f\left(\rho_{R}\right)\right)^{2}}}=\cos \left(\theta+2 \theta^{\prime}\left(\rho_{R}\right)\right), \\
& \frac{f\left(\rho_{T}\right)-h}{\sqrt{\rho_{T}^{2}+\left(f\left(\rho_{T}\right)-h\right)^{2}}}=\cos \theta,
\end{aligned}
$$

determined the parameters $\rho_{R}$ and $\rho_{T}$, which correspond to a certain angle $\theta$. The first, the second, and the third term of the sum in (10) are respectively an incident wave, a wave reflected from the reflector, and a wave transmitted through the reflector. The Heaviside step function $\chi(\theta)$ is equal to 1 or 0 in the regions of propagation or non-propagation, respectively, of the incident, reflected, and transmitted waves. The factors $s^{\text {inc }}$ and $s^{\text {refl }}$ are the eikonals of the incident and reflected waves, respectively. The components of the radiation pattern of the annular electric current on the edge of the reflector are the following:

$$
\begin{aligned}
& H_{\theta}^{s}(\theta, \varphi)=-2 \mathrm{R}_{\varphi}(L) H_{\varphi}\left(\theta^{i n c}, \varphi-\frac{\pi}{2 n}\right) \times \\
& \times \frac{e^{-i k L_{s e}}}{L_{s e}} \frac{i}{k} H_{\theta}^{\tau}(\theta, L) e^{i k f(L) \cos \theta}+ \\
& +2 \mathrm{R}_{\tau}(L) \cos \left(\theta^{i n c}+\theta^{\prime}(L)\right) H_{\theta}\left(\theta^{i n c}, \varphi\right) \times \\
& \times \frac{e^{-i k L_{s e}}}{L_{s e}} \frac{i}{k} H_{\theta}^{\varphi}(\theta, L) e^{i k f(L) \cos \theta}, \\
& H_{\varphi}^{s}(\theta, \varphi)=-2 \mathrm{R}_{\varphi}(L) H_{\varphi}\left(\theta^{i n c}, \varphi\right) \times \\
& \times \frac{e^{-i k L_{s e}}}{L_{s e}} \frac{i}{k} H_{\varphi}^{\tau}(\theta, L) e^{i k f(L) \cos \theta}+ \\
& +2 \mathrm{R}_{\tau}(L) \cos \left(\theta^{i n c}+\theta^{\prime}(L)\right) H_{\theta}\left(\theta^{i n c}, \varphi-\frac{\pi}{2 n}\right) \times \\
& \times \frac{e^{-i k L_{s e}}}{L_{s e}} \frac{i}{k} H_{\varphi}^{\varphi}(\theta, L) e^{i k f(L) \cos \theta},
\end{aligned}
$$

where the functions from (8) are calculated using the integral representation of the Bessel function (see formula (A.4) in the appendix) as follows:

$$
\begin{aligned}
& H_{\theta}^{\tau}(\theta, \rho)=-i^{n} \frac{k \rho}{4} \cos \left(\theta^{\prime}(\rho)\right) \times \\
& \times\left[\mathbf{J}_{n-1}(k \rho \sin \theta)+\mathbf{J}_{n+1}(k \rho \sin \theta)\right], \\
& H_{\theta}^{\varphi}(\theta, \rho)=i^{n} \frac{k \rho}{4}\left[\mathbf{J}_{n-1}(k \rho \sin \theta)-\mathbf{J}_{n+1}(k \rho \sin \theta)\right], \\
& H_{\varphi}^{\tau}(\theta, \rho)=-i^{n} \frac{k \rho}{4} \cos \theta \cos \left(\theta^{\prime}(\rho)\right) \times \\
& \times\left[\mathbf{J}_{n-1}(k \rho \sin \theta)-\mathbf{J}_{n+1}(k \rho \sin \theta)\right]+ \\
& +i^{n+1} \frac{k \rho}{2} \sin \theta \sin \left(\theta^{\prime}(\rho)\right) \mathbf{J}_{n}(k \rho \sin \theta), \\
& H_{\varphi}^{\varphi}(\theta, \rho)=-i^{n} \frac{k \rho}{4} \cos \theta \times \\
& \times\left[\mathbf{J}_{n-1}(k \rho \sin \theta)+\mathbf{J}_{n+1}(k \rho \sin \theta)\right] .
\end{aligned}
$$

\section{B. The Observation Angles Far from the Z-Axis}

For the interval of observation angles $\theta$ far from the $z$-axis, we initially applied the stationary phase method for the case when a stationary point was far from the end of the integration to the integrals in (8). Following this, we substituted the first term of the obtained asymptotic expansion into (7). To determine the two first terms of the asymptotic expansion of the integrals in (7), we used the stationary phase method for the case of the possible closeness of a stationary point to the end of the integration [22]. As a result, the asymptotic expression of the meridional (azimuthal) component of the radiation pattern for the observation angles $\theta$ far from the $z$-axis was calculated as follows:

$$
\begin{aligned}
& H_{\theta(\varphi)}^{P O}(\theta, \varphi)=H_{\theta(\varphi)}^{G O}(\theta, \varphi)+\sqrt{\frac{L}{\sin \theta \sqrt{h^{2}+L^{2}}}} \times \\
& \times\left[\left(1-\mathrm{T}_{\tau(\varphi)}(L)\right) H_{\theta(\varphi)}(\theta, \varphi) e^{i k \mathrm{~s}^{i n c}(\theta)} \times\right. \\
& \times\left(F^{r}(\theta)-\tilde{F}^{r}(\theta) \cos \left(\left(\theta^{i n c}-\theta\right) / 2\right)\right)+ \\
& +\xi_{\theta(\varphi)} \mathrm{R}_{\tau(\varphi)}(L) H_{\theta(\varphi)}\left(\pi-\left(\theta+2 \theta^{\prime}(L)\right), \varphi\right) e^{i k s^{r e f l}(\theta)} \times \\
& \left.\times\left(F^{m}(\theta)-\tilde{F}^{m}(\theta) \cos \left(\left(\theta^{r e f l}-\theta\right) / 2\right)\right)\right]+ \\
& +\sqrt{\frac{L}{\sin \theta \sqrt{h^{2}+L^{2}}}} H_{\theta(\varphi)}\left(\theta^{i n c}, \varphi\right) \times \\
& \times\left[\left(1-\mathrm{T}_{\tau(\varphi)}(L)\right) e^{i k \mathrm{~s}^{i n c}(\theta)} \tilde{F}^{r}(\theta) \cos \left(\left(\theta^{i n c}-\theta\right) / 2\right)+\right. \\
& \left.+\xi_{\theta(\varphi)} \mathrm{R}_{\tau(\varphi)}(L) e^{i k \mathrm{~s}^{r e f l}(\theta)} \tilde{F}^{m}(\theta) \cos \left(\left(\theta^{r e f l}-\theta\right) / 2\right)\right] \text {, }
\end{aligned}
$$

where 


$$
\begin{aligned}
& F^{r, m}(\theta)=F\left(s_{1}^{r, m}(\theta)\right)-(-1)^{n} i F\left(s_{2}^{r, m}(\theta)\right), \\
& \tilde{F}^{r, m}(\theta)=\tilde{F}\left(s_{1}^{r, m}(\theta)\right)-(-1)^{n} i \tilde{F}\left(s_{2}^{r, m}(\theta)\right), \\
& s_{1}^{r}(\theta)=\sqrt{2 k L_{s e}} \sin \left(\left(\theta^{i n c}-\theta\right) / 2\right), \\
& s_{1}^{m}(\theta)=\sqrt{2 k L_{s e}} \sin \left(\left(\theta^{r e f l}-\theta\right) / 2\right), \\
& s_{2}^{r, m}(\theta)= \begin{cases}s_{1}^{r, m}(-\theta), & 0 \leq \theta<\pi / 2 \\
s_{1}^{r, m}(2 \pi-\theta), & \pi / 2 \leq \theta \leq \pi\end{cases}
\end{aligned}
$$

and function $\tilde{F}(x)$ is the first term of the asymptotic expansion of the Fresnel integral $F(x)$ :

$$
\tilde{F}(x)=-\frac{e^{-i x^{2}}}{2 x \sqrt{i \pi}}, F(x)=-\operatorname{sgn}(x) \sqrt{\frac{i}{\pi}} \int_{-\infty}^{-s g n(x) x} e^{-i t^{2}} d t .
$$

\section{The Overlapping Sector of the Asymptotic Solutions}

The radiation patterns far from and near to the $z$-axis, which were obtained using the asymptotic formulae, overlapped in several sectors of angles $\theta$. The particular case of the scattering of a spherical wave created by a dipole on a semitransparent disk is investigated in [23]. The asymptotic formulae obtained in that paper are equivalent to (9) and (13). In [23], it was shown that the curves of radiation patterns calculated using the asymptotic formulae near to and far from the $z$-axis overlapped in several sectors of angles $\theta$. The overlapping region and the accuracy of the asymptotic formulae increase with the increase in the radius of the disk. Therefore, in the figures in Section IV
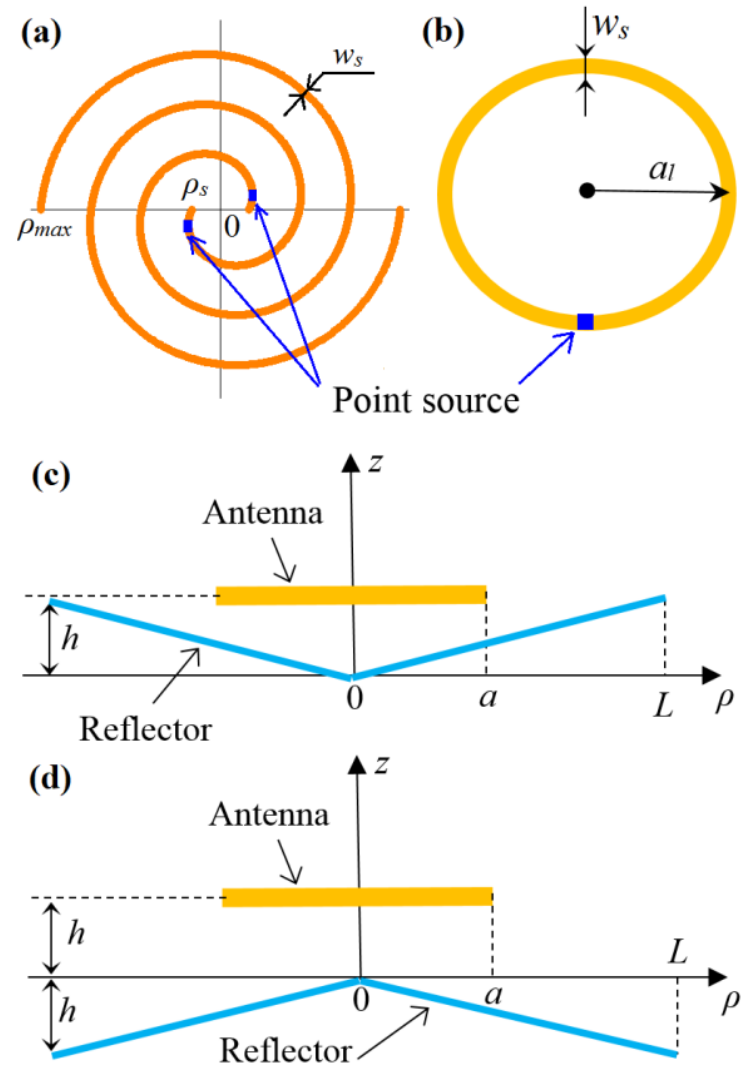

Fig. 3. The geometry of (a) the Archimedean spiral and (b) the loop antenna; antenna above (c) the concave and (d) convex corner reflector. of this paper, we plot a sole continuous curve through (9) and (13) when calculating the radiation patterns of the toroidal waves scattered by reflectors. The sole curve is based upon the overlapping of the radiation patterns near to and far from the $z$ axis.

\section{SPIRAL AND LOOP ANTENNAS WITH REFLECTORS}

As an example of the application of the asymptotic formulae, we investigated a plane with Archimedean two-wire spiral antenna working at the first harmonic radiation mode and a loop antenna above concave and convex reflectors (Fig. 3).

\section{A. The Modeling of the Antennas}

The Archimedean spiral antenna is made from infinitely thin strips of a width $w_{s}=0.01 \lambda$ (Fig. 3a). The shape of the antenna in the polar coordinates $(\rho, \varphi)$ is defined by the equation $\rho(\varphi)= \pm \rho_{s} \pm a_{s} \varphi /(2 \pi)$. To obtain the maximum radius of the spiral equal to $\rho_{\max }=0.22 \lambda$, we defined the start point $\rho_{s}=0.03 \lambda$ and the parameter $a_{s}=\rho_{\max }-\rho_{s}$. The number of the spiral's turns was 1.5. Each wire of the spiral was excited by a point source located near the start point of the antenna. We assumed that the antenna was perfectly matched. In [7], it is shown that the radiation pattern of the Archimedean spiral antenna in the case of the antiphase excitation of wires coincides with the radiation pattern of an annular azimuthal electric current of a traveling wave of radius $\lambda /(2 \pi)$ with the following distribution in the free space:

$\mathbf{j}_{e}^{\mathrm{o}}(\rho, \varphi, z)=\delta(\rho-\lambda /(2 \pi)) \delta(z) \mathrm{e}^{i \varphi} \boldsymbol{\varphi}_{0}$.

Here, $\delta(z)$ is the Dirac delta function. The radiation pattern of this azimuthal current was determined through the following components:

$$
\begin{aligned}
& \mathrm{H}_{\theta}(\theta, \varphi)=i \mathrm{e}^{i \varphi}\left(\mathrm{J}_{0}(\sin \theta)-\mathrm{J}_{2}(\sin \theta)\right), \\
& \mathrm{H}_{\varphi}(\theta, \varphi)=-\cos \theta \mathrm{e}^{i \varphi}\left(\mathrm{J}_{0}(\sin \theta)+\mathrm{J}_{2}(\sin \theta)\right) .
\end{aligned}
$$

In [2], it is shown that the electric current distribution of a loop antenna of a radius $a_{l}=\lambda /(2 \pi)$ made from an infinitely thin strip of a width $w_{s}$ (Fig. 3b), which is excited by a point source, can be approximated by the annular azimuthal electric current of a traveling wave $\mathbf{j}_{e}{ }^{\circ}$. Therefore, all the results obtained in Section IV for the spiral antenna are also applicable to the loop antenna.

\section{B. Back Radiation Suppression Using a Perfectly Conducting Reflector}

The spiral antenna was mounted on a reflector for the purpose of back radiation suppression. We firstly found the profile of a PEC reflector when $\mathrm{R}_{\tau}=\mathrm{R}_{\varphi}=1$ and $\mathrm{T}_{\tau}=\mathrm{T}_{\varphi}=0$, which are optimal for suppressing the back radiation. It follows from the asymptotic formula (9) that the back radiation near the $z$ axis is created by the annular electric current on the edge of the reflector induced by the source current $\mathbf{j}_{e}{ }^{\circ}$. To reduce the back radiation for a given radius of reflector $L$, we should reduce the magnitude of the excitation of the edge current. From formulae (15), we can see that $H_{\varphi}=0$ in the direction of $\theta=\pi / 2$, that is, the magnitude of the excitation of the current on the edge of the reflector is minimal when the edge of the reflector is located at 

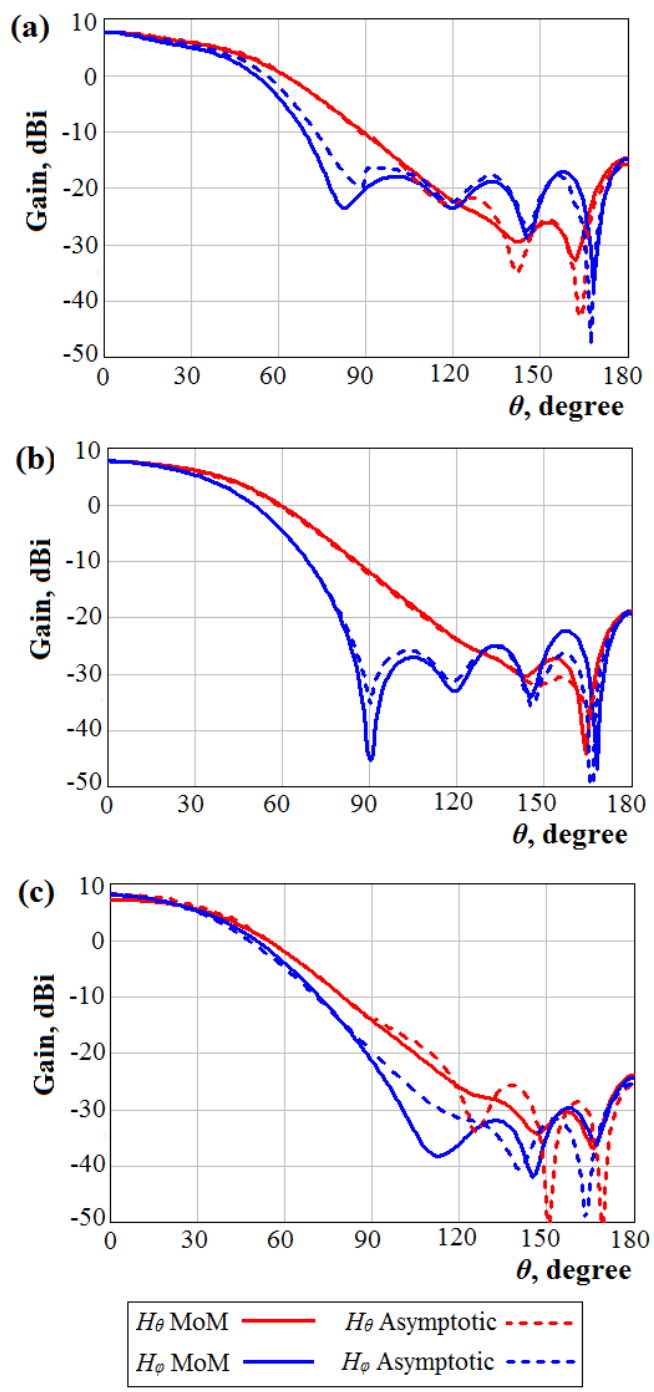

Fig. 4. The radiation patterns of the spiral antenna above (a) the convex, (b) the disk, and (c) the concave reflector.

the same height as the current $\mathbf{j}_{e}{ }^{\circ}(f(L)=h)$. It also follows from the asymptotic formula (11) that the magnitude of back radiation near the $z$-axis does not depend upon the reflector's shape $f(\rho)$ in this case. Therefore, a concave PEC corner reflector (Fig. 3c) is the easiest geometry required to obtain the back radiation suppression of the spiral antenna.

To prove that the placement of the spiral antenna at the level of the reflector's edge improves the front-to-back ratio (FBR), we plotted the radiation patterns of the antenna above concave, disk, and convex PEC reflectors of radiuses $L=1.5 \lambda$ on the distance $h=0.15 \lambda$ using the asymptotic formulae (9) and (13), and numerical simulations of the structures in EDEM software [24]. This software uses the MoM to simulate the scattering of electromagnetic waves created by antennas on arbitrarily shaped scatterers. Figure 4 shows the meridional and azimuthal components of the radiation patterns of the structures plotted using the asymptotic formulae and the numerical simulation. From this figure we can see that the asymptotic formulae allow the radiation patterns of the spiral antenna above the PEC reflectors to be calculated with a high level of accuracy. Some mismatches between the calculated and simulated results were observed near angle $\theta=90^{\circ}$ for the convex reflector and for angles $\theta>90^{\circ}$ for the concave reflector. The mismatch for the convex reflector can be explained by the fact that the asymptotic solution was based on the PO method, which assumes that the electric current is induced on the surface of the reflector according to the GO laws. In reality, the electric current has a more complex distribution; an additional current occurs near the apex of the reflector, which has the form of a wave that quickly decreases in relation to the distance from the apex. Therefore, the accuracy of the asymptotic formulae near the angle $\theta=90^{\circ}$ could be improved by adding the radiation pattern of the additional current, as well as taking into account one more term of the PO asymptotic expansion. The mismatch for the concave reflector appears at a level below $-25 \mathrm{~dB}$ from the maximum, which results in an error in the radiation pattern calculation of less than 5\%. The calculation error can be explained by the fact that the radiation pattern of the spiral antenna is not exactly the same as that of the current $\mathbf{j}_{e}{ }^{\circ}$. The FBRs of the structure were $22.4,26.6$, and $31.2 \mathrm{~dB}$ for the convex, disk, and concave reflectors, respectively. Thus, the taper of the reflector significantly influences the FBR.

\section{Back Radiation Suppression Using a Semitransparent Surface}

Further improvement of the FBR of the spiral antenna above the concave corner reflector is possible through the creation of a semitransparent edge. In [3,4] it is shown that the FBR of a patch antenna and a monopole with a semitransparent disk reflector is achieved through the use of a thin resistive layer based upon depositing carbon paste onto thin Kapton film. Such a resistive surface is characterized by a real isotropic impedance $Z_{\tau \tau}=Z_{\varphi \varphi}=Z_{S T}$ distributed uniformly at the edge of the reflector. Following the method of the aforementioned papers, we synthesized a semitransparent area with a uniform resistive isotropic impedance distribution at the edge of the reflector (Fig. 5). We assumed that the reflector had a central PEC area with a radius of $L_{S T}\left(Z_{S T}=0\right.$ when $\left.0 \leq \rho<L_{S T}\right)$, and a periphery area with the uniform isotropic resistive impedance distribution $Z_{S T}$ when $L_{S T} \leq \rho \leq L$. There was a discontinuity in the impedance distribution along the reflector's profile at the junction of the PEC semitransparent areas. The asymptotic formulae in Section III were obtained with the assumption of the slow varying of the impedance distribution along the reflector, hence, we could not directly apply the formulae for the synthesis of $Z_{S T}$. For the optimization of the FBR when the impedance distribution has a discontinuity, we can only use the asymptotic formula near the $z$-axis (9). The analysis of formula (9) shows that the radiation

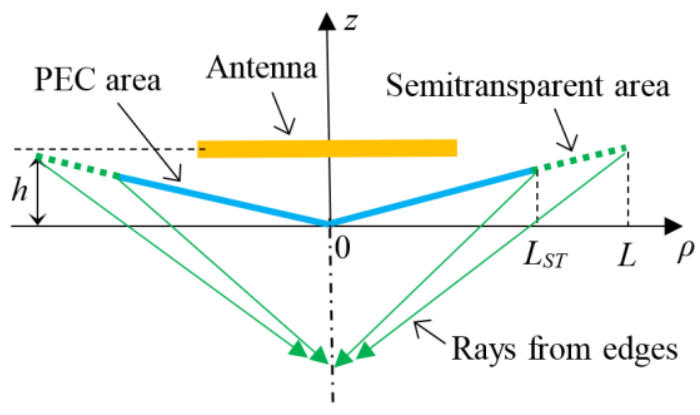

Fig. 5. The geometry of the reflector with the semitransparent edge and the ray propagation model. 
near the $z$-axis consisted of the GO pattern and the pattern created by the annular electric current on the edge of the reflector. When the central PEC area of the reflector is larger than the radius of the antenna $\left(L_{S T}>\rho_{\max }\right)$, the back radiation in the direction of $\theta=180^{\circ}$ is created only by the annular electric current on the edge of the reflector when $\rho=L$. In the considered case of the impedance distribution with the discontinuity, we assumed that the back radiation in the direction of $\theta=180^{\circ}$ was created by the annular electric current on the edge of the reflector and an annular electric current, which appears at the point of discontinuity when $\rho=L_{S T}$. Figure 5 shows a model of GO rays propagated in the direction of $\theta=180^{\circ}$ from these two annular electric currents. Thus, the asymptotic expansion of the total field at $\theta \rightarrow 180^{\circ}$ when the impedance distribution has the discontinuity was calculated as follows for the meridional (azimuthal) polarization of the incident wave:

$$
H_{\theta(\varphi)}^{P O}(\theta, \varphi)=H_{\theta(\varphi)}^{s}(\theta, \varphi)+H_{\theta(\varphi)}^{d}(\theta, \varphi) \text {. }
$$

Here, the first term of the sum was calculated using formula (11). The second term is the component of the radiation pattern of the annular electric current at the point of discontinuity:

$$
\begin{aligned}
& H_{\theta}^{d}(\theta, \varphi)=-2 \mathrm{R}^{d} H_{\varphi}\left(\theta_{d}^{i n c}, \varphi-\frac{\pi}{2 n}\right) \times \\
& \times \frac{e^{-i k L_{s e}^{d}}}{L_{s e}^{d}} \frac{i}{k} H_{\theta}^{\tau}\left(\theta, L_{S T}\right) e^{i k f\left(L_{S T}\right) \cos \theta}+ \\
& +2 \mathrm{R}^{d} \cos \left(\theta_{d}^{i n c}+\theta^{\prime}\left(L_{S T}\right)\right) H_{\theta}\left(\theta_{d}^{i n c}, \varphi\right) \times \\
& \times \frac{e^{-i k L_{s e}^{d}}}{L_{s e}^{d}} \frac{i}{k} H_{\theta}^{\varphi}\left(\theta, L_{S T}\right) e^{i k f\left(L_{S T}\right) \cos \theta} \\
& H_{\varphi}^{d}(\theta, \varphi)=-2 \mathrm{R}^{d} H_{\varphi}\left(\theta_{d}^{i n c}, \varphi\right) \times \\
& \times \frac{e^{-i k L_{s e}^{d}}}{L_{s e}^{d}} \frac{i}{k} H_{\varphi}^{\tau}\left(\theta, L_{S T}\right) e^{i k f\left(L_{S T}\right) \cos \theta}+ \\
& +2 \mathrm{R}^{d} \cos \left(\theta_{d}^{i n c}+\theta^{\prime}\left(L_{S T}\right)\right) H_{\theta}\left(\theta_{d}^{i n c}, \varphi-\frac{\pi}{2 n}\right) \times \\
& \times \frac{e^{-i k L_{s e}^{d}}}{L_{s e}^{d}} \frac{i}{k} H_{\varphi}^{\varphi}\left(\theta, L_{S T}\right) e^{i k f\left(L_{S T}\right) \cos \theta},
\end{aligned}
$$

where

$$
L_{s e}^{d}=\sqrt{L_{S T}^{2}+\left(h-f\left(L_{S T}\right)\right)^{2}}, \theta_{d}^{i n c}=\arccos \left(\frac{f\left(L_{S T}\right)-h}{L_{s e}^{d}}\right) .
$$

The value for the reflection coefficient at the point of discontinuity $\mathrm{R}^{d}$ was taken from the approximate solution of the scattering by a junction of PEC and semitransparent half-planes [25] and was calculated as $\mathrm{R}^{d}=1-Z_{0} /\left(Z_{S T}+Z_{0}\right)$. Figure 6 shows the back radiation suppression in the direction of $\theta=180^{\circ}$ for the concave semitransparent corner reflector of the radius $L=1.5 \lambda$ as compared with the PEC reflector of the same radius with different values for the impedance $Z_{S T}$ and radius $L_{S T}$. From Fig. 6 , we can see that for the improvement of the back radiation suppression, parameters $L_{S T}$ and $Z_{S T}$ should be within the range

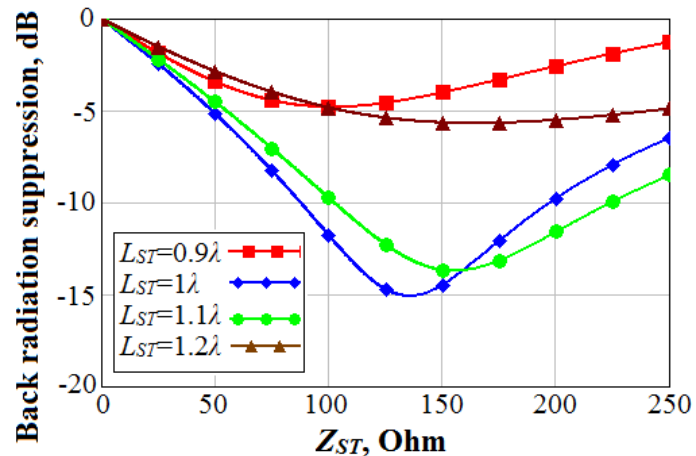

Fig. 6. The dependence of the back radiation suppression on the impedance of the semitransparent area for different radii of the PEC area.

of $1 \lambda-1.1 \lambda$ and $120-170 \Omega$, respectively. The best back radiation suppression was observed for parameters $L_{S T}=1 \lambda$ and $Z_{S T}=140$ $\Omega$. However, due to the previously mentioned accuracy of the PO method and the approximation of the spiral antenna radiation pattern, the maximum back radiation suppression of the antenna with the semitransparent reflector was observed when $L_{S T}=1.1 \lambda$ and $Z_{S T}=130 \Omega$, as an additional analysis using numerical simulations demonstrated. The radiation patterns of the spiral antenna above the concave semitransparent corner reflector with the parameters $L_{S T}=1.1 \lambda$ and $Z_{S T}=130 \Omega$, which

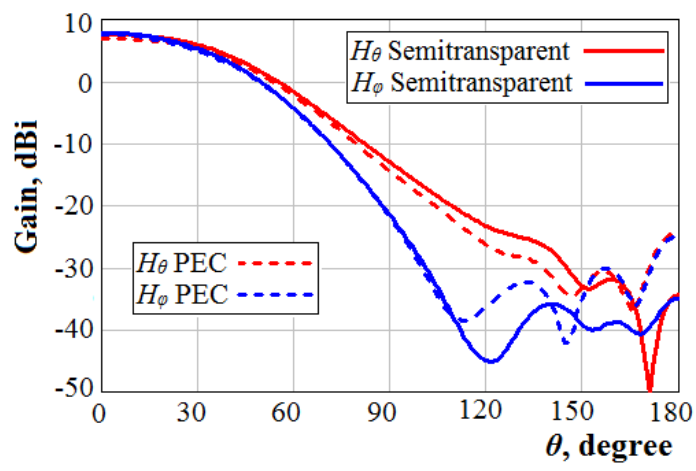

Fig. 7. The radiation patterns of the spiral antenna above the PEC and concave semitransparent corner reflectors.

were obtained using a numerical simulation of the structure in EDEM software, are shown in Fig. 7. From this figure, we can see that the simulated FBR for the semitransparent reflector is $41.9 \mathrm{~dB}$. The improvement in back radiation suppression is 10.7 $\mathrm{dB}$ as compared with the concave PEC corner reflector. The peak gain of the antenna was 7.65 and $7.89 \mathrm{dBi}$ for the PEC and semitransparent reflectors, respectively. Therefore, the back radiation suppression appears to be due to the interference of the fields created by the electric current on the reflector's surface but not due to any energy loss in the resistive surface. Such interference leads to the small peak gain increasing. The simulated FBR and the one calculated using formula (16) were 41.9 and $46.4 \mathrm{~dB}$, respectively, which correspond to the back radiation values of 0.008 and 0.0048 for a normalized radiation pattern. Thus, the proposed asymptotic formula (16) provides a calculation of the radiation pattern at $\theta=180^{\circ}$ with an error of less than $1 \%$. 

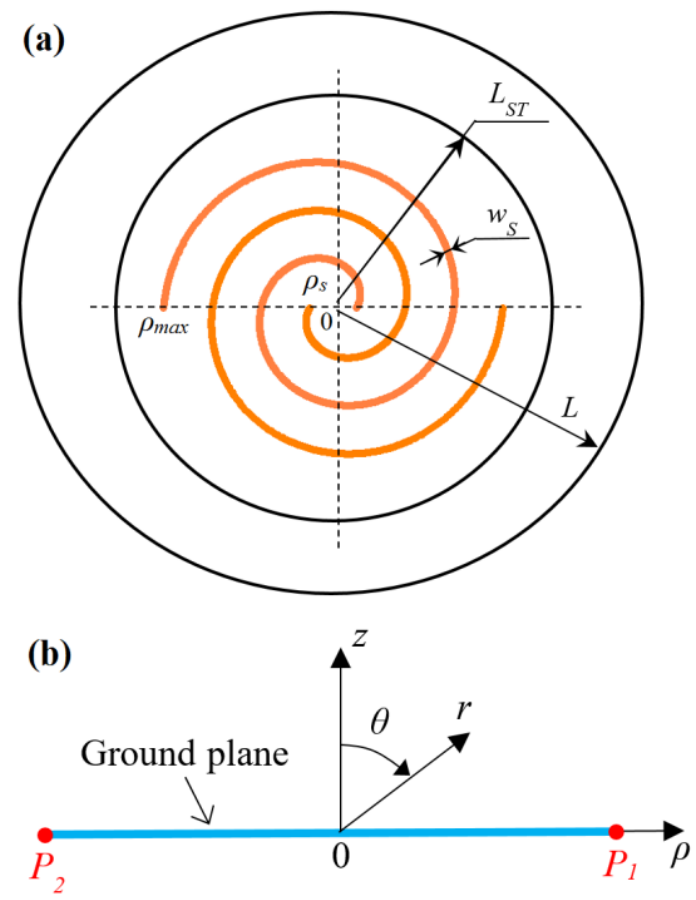

Fig. 8. (a) the geometry of the Archimedean slot spiral antenna with the ground plane; (b) the determination of the second order diffraction field.

\section{Slot Spiral Antenna With A Ground Plane}

In this section, we investigate the radiation patterns of an Archimedean slot spiral antenna with PEC and semitransparent ground planes. We consider the antenna of the shape $\rho(\varphi)= \pm \rho_{s} \pm a_{s} \varphi /(2 \pi)$ made as a slot of a width $w_{s}$ on a disk ground plane as shown in Fig. 8. We assumed that the ground plane had a central PEC area with a radius of $L_{S T}\left(Z_{S T}=0\right.$ when $\left.0 \leq \rho<L_{S T}\right)$, and a periphery semitransparent area with the isotropic impedance $Z_{S T}$ when $L_{S T} \leq \rho \leq L$. The number of the spiral's turns was 1.5 . Then, by analogy with the two-wire spiral antenna, the radiation pattern of the Archimedean slot spiral antenna located on an infinite PEC ground plane in the case of the antiphase excitation of the slots coincides with the radiation pattern of an annular azimuthal magnetic current of a traveling wave of radius $\lambda /(2 \pi)$ with the following distribution in free space:

$\mathbf{j}_{m}^{\mathrm{o}}(\rho, \varphi, z)=\delta(\rho-\lambda /(2 \pi)) \delta(z) \mathrm{e}^{i \varphi} \boldsymbol{\varphi}_{0}$.

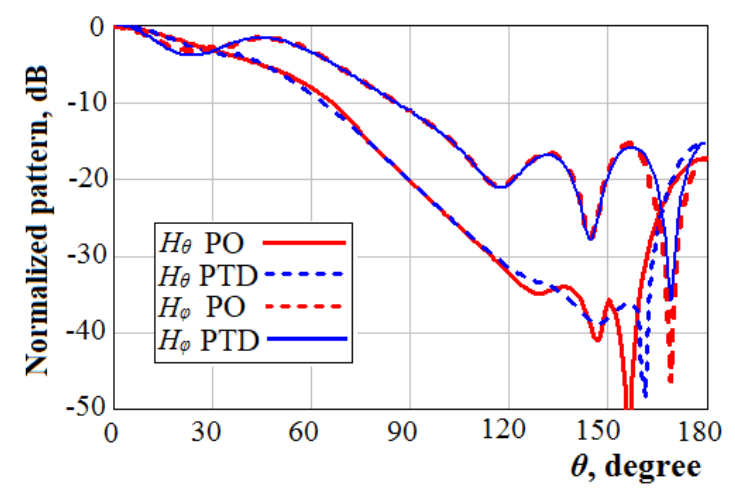

Fig. 9. The radiation patterns of the slot spiral antenna with the PEC ground plane.
The radiation pattern of this azimuthal current was determined through the following components:

$$
\begin{aligned}
& \mathrm{H}_{\theta}(\theta, \varphi)=-\cos \theta \mathrm{e}^{i \varphi}\left(\mathrm{J}_{0}(\sin \theta)+\mathrm{J}_{2}(\sin \theta)\right), \\
& \mathrm{H}_{\varphi}(\theta, \varphi)=-i \mathrm{e}^{i \varphi}\left(\mathrm{J}_{0}(\sin \theta)-\mathrm{J}_{2}(\sin \theta)\right) .
\end{aligned}
$$

Figure 9 shows the normalized meridional and azimuthal components of the radiation pattern of the annular magnetic current on a PEC ground plane of radius $L=1.5 \lambda$. The radiation patterns in Fig. 9 were plotted using the asymptotic formulae (9) and (13) in the PO approximation, as well as some asymptotic formulae from [15] obtained in the physical theory of diffraction (PTD) approximation for the scattering problem under study. As shown in [15], the PTD approximation provides an accuracy of less than $2 \%$ as compared with the rigorous solution of the scattering problem for disk reflectors of radius $L>\lambda$. Thus, we used the PTD approximation to estimate the accuracy of the PO approximation. We noted that a small discontinuity appears at $\theta=90^{\circ}$ for the curve of the azimuthal component of the radiation pattern in the PO approximation plotted using asymptotic formula (13). This discontinuity appears because in (13) we used only the first two terms of the asymptotic expansion of the integrals in (7). The discontinuity was smoothed by introducing one more term of the asymptotic expansion $H_{\varphi}{ }^{2 d}$, which describes a second order diffraction field. This field emanates from point $P_{1}$ due to its illumination by the field scattered at $P_{2}$, as shown in Fig. 8b. The term $H_{\varphi}{ }^{2 d}$ was calculated as follows for the disk ground plane:

$$
\begin{aligned}
& H_{\varphi}^{2 d}(\theta, \varphi)=\left(\mathrm{R}_{\varphi}(L)\right)^{2} H_{\varphi}\left(\theta^{i n c}, \varphi\right) \frac{(-1)^{n} \sqrt{2 i L}}{L_{s e} \sqrt{\pi k \sin \theta}} \times \\
& \times \operatorname{sgn}\left(\sin \left(\frac{\pi}{4}-\frac{\theta}{2}\right)\right) F(\sqrt{2 k L(1-\sin \theta)}) e^{-i k\left(L \sin \theta+L_{s e}\right)} .
\end{aligned}
$$

The curve of the azimuthal component of the radiation pattern in the PO approximation in Fig. 9 was plotted taking (20) into account. From Fig. 9, we can see that the curves obtained using the two methods coincide for most of the observation angles. The main difference is observed for $\theta>150^{\circ}$ at the level below $25 \mathrm{~dB}$, which corresponds to a difference in the patterns of less than 5\%. The FBRs were $17.2 \mathrm{~dB}$ and $15.1 \mathrm{~dB}$ for the PO and PTD approximations, respectively, which led to a $2.1 \mathrm{~dB}$ FBR difference. This result corresponds with the result from [2],

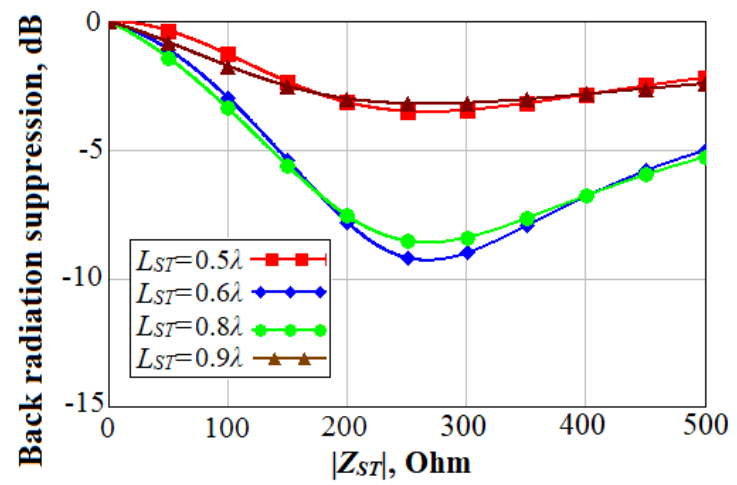

Fig. 10. The dependence of the back radiation suppression on the modulus of the impedance of the semitransparent area for different radii of the PEC area. 
where it was shown that the PO method provides an error of approximately $2 \mathrm{~dB}$ in the calculation of the back radiation for disk reflectors of radii more than $\lambda$.

It was shown in $[4,15]$ that the magnitude of the back radiation of the annular magnetic current with the first azimuthal harmonic (18) located on a disk PEC ground plane is constant when the ground plane's radius tends to infinity. Therefore, the FBR of the Archimedean slot spiral antenna cannot be improved by increasing the radius of the ground plane when it tends to infinity. To improve the FBR, we used a semitransparent surface with a capacitive isotropic impedance $Z_{S T}$ distributed uniformly at the edge of the reflector. The capacitive character of the impedance assumes that the argument of $Z_{S T}$ is equal to $-\pi / 2$. Such a capacitive semitransparent surface can be implemented, for example, through perforated grids of slots on the PEC surface. Figure 10 shows the back radiation suppression in the direction of $\theta=180^{\circ}$ for the semitransparent ground plane of radius $L=1.5 \lambda$ as compared with the PEC one. The curves in Fig. 10 are plotted for different values of the impedance modulus $\left|Z_{S T}\right|$ and radius $L_{S T}$. From this figure, we can see that for the improvement of the back radiation suppression, parameters $L_{S T}$ and $\left|Z_{S T}\right|$ should be within the range of $0.6 \lambda-0.8 \lambda$ and $200-350 \Omega$, respectively.

\section{CONCLUSION}

We obtained the asymptotic formulae for the calculation of the radiation pattern of a toroidal (particularly spherical) wave scattered by a semitransparent reflector of revolution using the PO method. We used the asymptotic formulae to calculate the radiation patterns of the Archimedean two-wire spiral antenna and the loop antenna located above the concave and convex PEC corner reflectors. It was demonstrated that the placement of the antennas at the level of the edge of the concave PEC reflectors improved the FBRs of the antennas. Additional back radiation suppression can be obtained using a resistive semitransparent surface at the reflector's edge. For the Archimedean slot spiral antenna with the ground plane, back radiation suppression can be obtained using a capacitive semitransparent surface at the ground plane's edge.

\section{APPENDIX}

A ring of radial electric $\mathbf{j}_{e}{ }^{\rho}$ or magnetic $\mathbf{j}_{m}{ }^{\rho}$ current of radius $a$ in free space has the following form:

$$
\mathbf{j}_{e(m)}^{\rho}(\rho, \varphi, z)=I_{e(m)}^{\rho} \delta(\rho-a) \delta(z) \cos (n \varphi+\psi) \boldsymbol{\rho}_{0},
$$

where $I_{e}{ }^{\rho}$ and $I_{m}{ }^{\rho}$ is the magnitude of the radial electric and magnetic currents, respectively. The meridional and azimuthal components of the radiation pattern of the ring of radial current are calculated through the expressions:

$$
\begin{aligned}
& \mathrm{H}_{\theta}(\theta, \varphi)=-i^{n} \frac{I_{e}^{\rho} k a}{4} \sin (n \varphi+\psi) \times \\
& \times\left[\mathrm{J}_{n-1}(k a \sin \theta)+\mathbf{J}_{n+1}(k a \sin \theta)\right], \\
& \mathrm{H}_{\varphi}(\theta, \varphi)=-i^{n} \frac{I_{e}^{\rho} k a}{4} \cos \theta \cos (n \varphi+\psi) \times \\
& \times\left[\mathbf{J}_{n-1}(k a \sin \theta)-\mathbf{J}_{n+1}(k a \sin \theta)\right],
\end{aligned}
$$

for radial electric current, and

$$
\begin{aligned}
& \mathrm{H}_{\theta}(\theta, \varphi)=-i^{n} \frac{I_{m}^{\rho} k a}{4 Z_{0}} \cos \theta \cos (n \varphi+\psi) \times \\
& \times\left[\mathbf{J}_{n-1}(k a \sin \theta)-\mathbf{J}_{n+1}(k a \sin \theta)\right], \\
& \mathrm{H}_{\varphi}(\theta, \varphi)=i^{n} \frac{I_{m}^{\rho} k a}{4 Z_{0}} \sin (n \varphi+\psi) \times \\
& \times\left[\mathbf{J}_{n-1}(k a \sin \theta)+\mathbf{J}_{n+1}(k a \sin \theta)\right],
\end{aligned}
$$

for radial magnetic current, where the Bessel function has the following integral representation:

$$
\mathbf{J}_{n}(z)=\frac{1}{2 \pi} \int_{-\pi}^{\pi} e^{i\left(z \sin \phi^{\prime}-n \phi^{\prime}\right)} d \phi^{\prime}
$$

A ring of azimuthal electric $\mathbf{j}_{e}{ }^{\varphi}$ or magnetic $\mathbf{j}_{m}{ }^{\varphi}$ current of radius $a$ in free space has the following form:

$$
\mathbf{j}_{e(m)}^{\varphi}(\rho, \varphi, z)=I_{e(m)}^{\varphi} \delta(\rho-a) \delta(z) \cos (n \varphi+\psi) \boldsymbol{\varphi}_{0},
$$

where $I_{e}{ }^{\varphi}$ and $I_{m}{ }^{\varphi}$ is the magnitude of the azimuthal electric and magnetic currents, respectively. The meridional and azimuthal components of the radiation pattern of the ring of azimuthal current are calculated through the expressions:

$$
\begin{aligned}
& \mathrm{H}_{\theta}(\theta, \varphi)=i^{n} \frac{I_{e}^{\varphi} k a}{4} \cos (n \varphi+\psi) \times \\
& \times\left[\mathbf{J}_{n-1}(k a \sin \theta)-\mathbf{J}_{n+1}(k a \sin \theta)\right], \\
& \mathbf{H}_{\varphi}(\theta, \varphi)=-i^{n} \frac{I_{e}^{\varphi} k a}{4} \cos \theta \sin (n \varphi+\psi) \times \\
& \times\left[\mathbf{J}_{n-1}(k a \sin \theta)+\mathbf{J}_{n+1}(k a \sin \theta)\right],
\end{aligned}
$$

for the azimuthal electric current, and

$$
\begin{aligned}
& \mathrm{H}_{\theta}(\theta, \varphi)=-i^{n} \frac{I_{m}^{\varphi} k a}{4 Z_{0}} \cos \theta \sin (n \varphi+\psi) \times \\
& \times\left[\mathbf{J}_{n-1}(k a \sin \theta)+\mathbf{J}_{n+1}(k a \sin \theta)\right], \\
& \mathrm{H}_{\varphi}(\theta, \varphi)=-i^{n} \frac{I_{m}^{\varphi} k a}{4 Z_{0}} \cos (n \varphi+\psi) \times \\
& \times\left[\mathbf{J}_{n-1}(k a \sin \theta)-\mathbf{J}_{n+1}(k a \sin \theta)\right],
\end{aligned}
$$

for the azimuthal magnetic current. A ring of axial electric $\mathbf{j}_{e}^{z}$ 
or magnetic $\mathbf{j}_{m}{ }^{z}$ current of radius $a$ in free space has the following form:

$$
\mathbf{j}_{e(m)}^{z}(\rho, \varphi, z)=I_{e(m)}^{z} \delta(\rho-a) \delta(z) \cos (n \varphi+\psi) \mathbf{z}_{0},
$$

where $I_{e}{ }^{z}$ and $I_{m}{ }^{z}$ is the magnitude of the axial electric and magnetic currents, respectively. The meridional and azimuthal components of the radiation pattern of the ring of axial current are calculated through the expressions:

$$
\begin{aligned}
& \mathrm{H}_{\theta}=0, \\
& \mathrm{H}_{\varphi}(\theta, \varphi)=i^{n+1} \frac{I_{e}^{z} k a}{2} \sin \theta \cos (n \varphi+\psi) \mathrm{J}_{n}(k a \sin \theta),
\end{aligned}
$$

for axial electric current, and

$$
\begin{aligned}
& \mathrm{H}_{\theta}(\theta, \varphi)=i^{n+1} \frac{I_{m}^{z} k a}{2 Z_{0}} \sin \theta \cos (n \varphi+\psi) \mathrm{J}_{n}(k a \sin \theta) \\
& \mathrm{H}_{\varphi}=0
\end{aligned}
$$

for axial magnetic current.

\section{ACKNOWLEDGMENTS}

The author expresses his deep gratitude to the author of the EDEM software package, Dr. Alexander Davydov, for the free ultimate version of the software.

\section{REFERENCES}

[1] K. Klionovski, "Theoretical and Experimental Research of Diffraction on Round Semitransparent Ground Plane," IEEE Trans. Antennas Propag., vol. 61, no. 6, pp. 3207-3215, June 2013.

[2] V. Kaloshin and K. Klionovski, "On Radiation of Omnidirectional Axisymmetric Antennas with Circular Ground Planes," Journal of Communications Technology and Electronics, vol. 60, no. 10, pp. 10621071, Oct. 2015.

[3] K. Klionovski, M. F. Farooqui and A. Shamim, "Back Radiation Suppression through a Semi-Transparent Round Ground Plane for a mmWave Monopole Antenna," in Proc. Antennas Propag. Soc. Int. Symp., San Diego, CA, July 2017, pp. 2583-2584.

[4] K. Klionovski and A. Shamim, "Back Radiation Suppression through a Semitransparent Ground Plane for a Millimeter-Wave Patch Antenna," IEEE Trans. Antennas Propag., vol. 65, no. 8, pp. 3935-3941, Aug. 2017.

[5] M. S. A. Mahmoud, T.-H. Lee, and W. D. Burnside, "Enhanced Compact Range Reflector Concept Using an R-Card Fence: Two-Dimensional Case," IEEE Trans. Antennas Propag., vol. 49, no. 3, pp. 419-428, March 2001.

[6] R. L. Haupt, "Adaptive reflector antenna using smart resistive sheets," Electron. Lett., vol. 42, no. 22, pp. 1257-1258, Oct. 2006.

[7] J. A. Kaiser, "Spiral antennas applied to scanning arrays," in Proc. Electronic Scanning Symposium, AFCRC and RADC, Cambridge, MA, Apr. 1958.

[8] D. R. Jackson, "Microstrip antennas," in Antenna engineering handbook. Fourth edition (ed. J. Volakis). New York, NY, USA: McGraw Hill Companies. 2007.

[9] T. S. Bird and A. W. Love, "Horn antennas," in Antenna engineering handbook. Fourth edition (ed. J. Volakis). New York, NY, USA: McGraw Hill Companies. 2007.

[10] J. Meixner, "The radiation pattern and induced current in a circular antenna with an annular slit", IRE Trans. Antennas Propag., vol. 4, no. 3. pp. 408411, July 1956
[11] Yu. V. Pimenov and Ye. V. Smirnov, "Excitation of an ideally conducting disc by a narrow annular slot", Radio Engineering and Electronic Physics, vol. 22, no. 5, pp. 5-11, 1977.

[12] M. I. Kontorovich and Yu. N. Novikov, "The effect of a screen on the performance of travelling-wave loop antennas", Radio Engineering and Electronic Physics, vol. 28, no. 9, pp. 26-31, 1983.

[13] M. I. Kontorovich and Yu. N. Novikov, "Diffraction of electromagnetic waves by a ring and disk", Soviet Physics. Technical Physics, vol. 29, pp. 1225-1227, Nov. 1984.

[14] M. I. Kontorovich and Yu. N. Novikov, "An iterative method of solving certain electrodynamic problems", USSR Computational Mathematics and Mathematical Physics, vol. 27, no. 1, pp. 94-97, May 1988.

[15] V. Kaloshin and K. Klionovski, "Scattering of the Field of a Ring Current by a Perfectly Conducting Disk," Journal of Communications Technology and Electronics, vol. 59, no. 6, pp. 481-495, June 2014.

[16] B. Ye. Kinber, "Diffraction of electromagnetic waves on a concave spherical surface", Radio Engineering and Electronic Physics, vol. 6, no. 10, pp. 1474-1479, 1961.

[17] O. P. Ponomarev, "Diffraction of Electromagnetic Waves by Concave Circumferential Surfaces: Application for Hybrid Reflector Antennas," Bulletin of the Russian Academy of Sciences: Physics, vol. 72, no. 12, pp. 1666-1670, Dec. 2008.

[18] O. Ponomarev, "The Analysis of Hybrid Reflector Antennas and Diffraction Antenna Arrays on the Basis of Surfaces with a Circular Profile," Wave Propagation (ed. A. Petrin), pp. 285-308, Rijeka, Croatia: IntechOpen. 2011.

[19] F. Oberhettinger and R. F. Dressler, "Electromagnetic fields in the presence of ideally conducting conical structures", Journal of Applied Mathematics and Physics, vol. 22, no. 5, pp. 937-950, Sept. 1971.

[20] V. A. Borovikov and B. Ye. Kinber, Geometrical Theory of Diffraction. London, UK: IEE, 1994.

[21] L. B. Felsen and N. Marcuvitz, Radiation and Scattering of Waves. Hoboken, NJ, USA: Wiley, 2003.

[22] R. M. Lewis, "Asymptotic theory of transients," in Electromagnetic Wave Theory. Part 2 (ed. J. Brown), pp. 845-869, New York, NY, USA: Pergamon press. 1967.

[23] V. Kaloshin and K. Klionovski, "Radiation of a dipole located on axis of a semitransparent disk," Journal of Radio Electronics, no. 5, May 2014, online available: http://jre.cplire.ru/jre/may14/10/text.pdf.

[24] http://edem3d.ru/

[25] T. B. A. Senior and J. L. Volakis, Approximate boundary conditions in electromagnetics. London, UK: The Institution of Electrical Engineers, 1995.

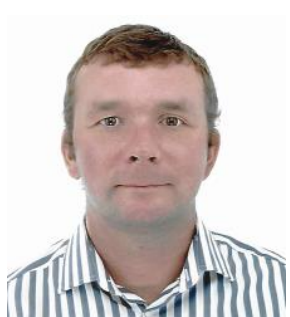

Kirill Klionovski was born in Krasnogorsk, Russia. He received the Engineer degree (Hons.) in electrical engineering from Moscow Aviation Institute, Moscow, Russia, in 2010, and the $\mathrm{Ph} . \mathrm{D}$. degree in radiophysics from the Kotel'nikov Institute of Radioengineering and Electronics, Russian Academy of Sciences, Moscow, Russia, in 2015.

His current research interests include asymptotic methods in applied electromagnetics. 